\title{
Performance of the BRIL luminometers at CMS in Run 2
}

\author{
O. Karacheban* on behalf of the CMS Collaboration \\ CERN, Geneva, Switzerland \\ E-mail: olena.karacheban@cern.ch
}

CMS features three luminosity subdetectors capable of providing real-time ("online") luminosity on a bunch-by-bunch level independently of the main CMS data acquisition system: the Fast Beam Conditions Monitor (BCM1F), the Hadron Forward (HF) calorimeter, and the Pixel Luminosity Telescope (PLT). These luminometers have operated since the beginning of Run 2 (2015 2018) at the LHC. In order to obtain an accurate luminosity measurement, we use van der Meer ( $v d M)$ scans to provide the absolute calibration, whereas corrections for effects such as efficiency loss due to radiation damage, nonlinear effects at high instantaneous luminosity, or effects due to the bunch train structure of the beams, are measured and subsequently applied. The calibration of the online luminosity subdetectors, the applied corrections, and comparisons with offline measurements using the pixel cluster counting (PCC) method, the radiation monitoring system (RAMSES) and muon system Drift Tubes (DT) are covered.

The European Physical Society Conference on High Energy Physics (EPS-HEP)

10-17 July 2019

Ghent, Belgium

${ }^{*}$ Speaker. 


\title{
1. Calibration of the CMS online luminometers using vdM scan technique
}
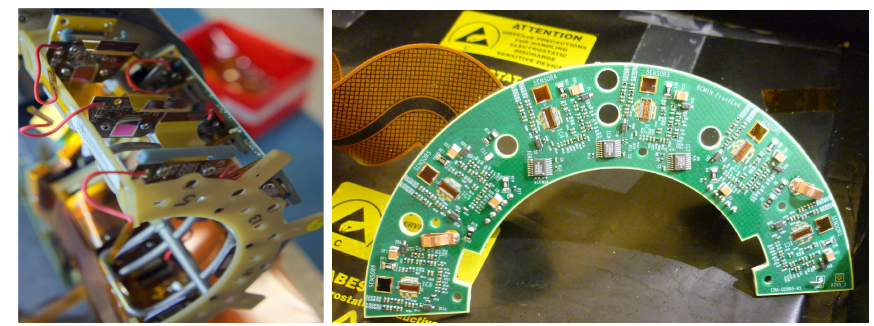

\author{
HF wedge
}

36 wedges in

total

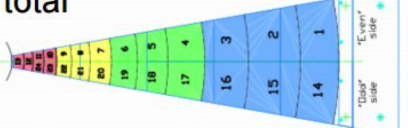

Figure 1: One quarter of the PLT detector (left), one quarter of the BCM1F detector (middle) and one section of the Hadronic Forward calorimeter (right).

Multiple luminometers are required to guaranty a reliable uninterrupted luminosity measurement and redundancy. CMS is equipped with dedicated online bunch by bunch luminosity detectors: the Pixel Luminosity Telescope (PLT [1, 2], Fig. 1 (left)) and the Fast Beam Condition Monitor (BCM1F [2, 3], Fig. 1 (middle)). Also other CMS subdetectors are used for luminosity, e.g. two rings of towers of the Forward Hadronic calorimeter HF [4] (one section is shown in Fig. 1 (right)), which provides luminosity measurement based on occupancy (HFOC) and sum of transverse energy (HFET). In addition, for an offline luminosity measurement, the CMS pixel detector and the radiation monitoring systems (RAMSES) and muon system Drift Tubes (DT) are used [4]. For the absolute luminosity calibration the van der Meer scan technique [4] is used at LHC. The vdM program is carried out once per year per collision mode (e.g. pp $13 \mathrm{TeV}$, pp $5 \mathrm{TeV}$, $\mathrm{PbPb}, \mathrm{pPb}$ ). For reproducibility and controlling the systematic uncertainty, several vdM scans are performed sequentially. The sketch of the beam positions during vdM scans in $\mathrm{X}$ and $\mathrm{Y}$ planes together with the detector rate as a function of beam separation are shown in Fig. 2 on the left. An example of the measured detector rate as a function of the beam separation is shown in Fig. 2 on the right with a fit of a single Gaussian function. The peak rates in $\mathrm{X}\left(R_{\max , \mathrm{X}}\right)$ and $\mathrm{Y}\left(R_{\max , \mathrm{Y}}\right)$ and the effective beam overlap widths in $\mathrm{X}\left(\Sigma_{X}\right)$ and $\mathrm{Y}\left(\Sigma_{Y}\right)$ are obtained from the fit, used to calculate the visible cross section $\left(\sigma_{\mathrm{vis}}\right)$ :

$$
\sigma_{v i s}=\pi \Sigma_{X} \Sigma_{Y}\left(R_{\max , \mathrm{X}}+R_{\max , \mathrm{Y}}\right)
$$

The measured $\sigma_{v i s}$ is a property of the luminometer and is used for the absolute luminosity calibration. $\sigma_{v i s}$ is measured per bunch crossing (BCID) and then averaged over all bunch crossings and $\mathrm{vdM}$ scans in the vdM program to obtain an absolute calibration per detector $\left(\sigma_{\mathrm{vis}, \mathrm{vdM}}\right)$, as described in detail in Ref. [4]. Luminosity is derived using detector rate $R$ and the final $\sigma_{\mathrm{vis}, \mathrm{vdM}}$ as:

$$
L=R / \sigma_{\mathrm{vis}, \mathrm{vdM}}
$$

\section{From vdM scans to LHC Run 2 operation}

To study rare physics processes we need to accumulate statistically significant data sets for precise physics cross section measurements. To fulfill this requirement LHC has to reach as high as possible integrated luminosity. Therefore, high bunch intensity, number of bunches, and the 

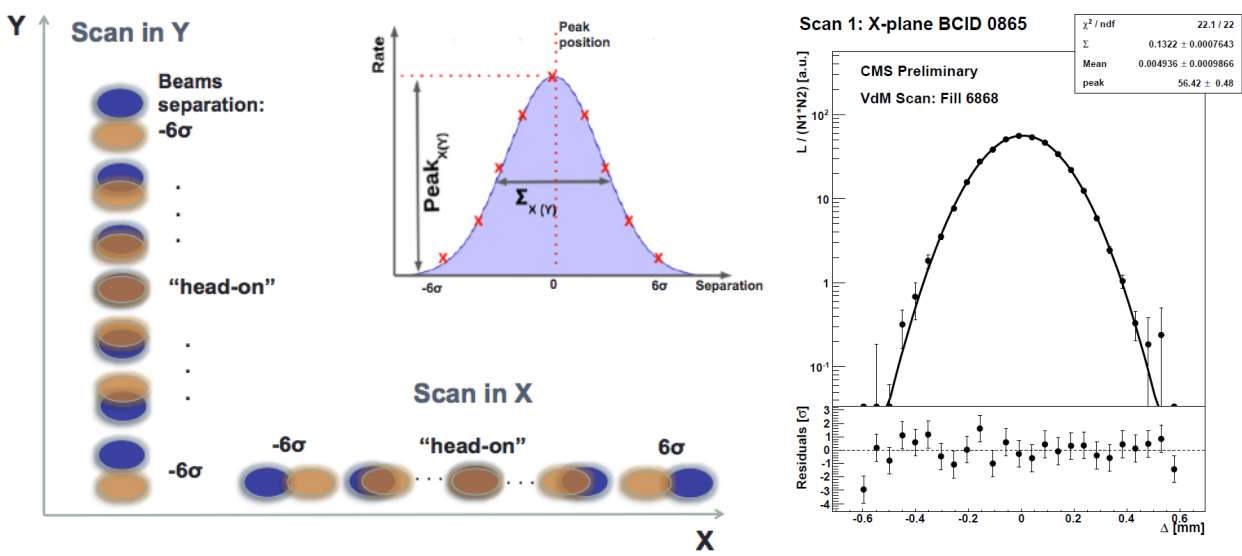

Figure 2: The sketch of a vdM scan in $\mathrm{X}$ and $\mathrm{Y}$ planes (left). Example of a real vdM scan (right) in $\mathrm{X}$ plane. Points represent the measured rate normalised to the beam current and line is a single Gaussian fit.

smallest possible beam sizes (emittance) are used in standard operation. As bunches are grouped into bunch trains and bunches collide with a crossing angle at the interaction points, additional effects, such as orbit effects, long-range beam-beam interactions, afterglow (spill over from one bunch crossing to the following), etc., have to be taken into account. It is important to mention that CMS online luminometers are equipped with $40 \mathrm{MHz}$ readout systems, without which it would not be possible to derive the per bunch crossing corrections listed above. Table 1 summarises the main differences affecting the luminosity measurement when changing the beam parameters from the ones used in the special vdM calibration fill to the ones used in the standard LHC operation.

\begin{tabular}{|l||l|l|}
\hline & vdM scan & LHC standard operation \\
\hline Filling scheme & Solo bunches $(30-130)$ & Bunch trains (up to 2500) \\
\hline Crossing angle & $\alpha / 2=0$ & in range $\alpha / 2=130-160 \mu \mathrm{rad}$ \\
\hline Beam size & $\sim 100 \mu \mathrm{m}\left(\beta^{*}=19 \mathrm{~m}\right)$ & $\sim 10-15 \mu \mathrm{m}\left(\beta^{*}=25-30 \mathrm{~cm}\right)$ \\
\hline Peak pileup & $\sim 0.5$ & $\sim 60$ \\
\hline
\end{tabular}

Table 1: Comparison of the beam conditions in the vdM calibration and standard LHC operation.

From 2017 onwards CMS performed emittance scans in almost every physics fill, these are similar to vdM scans but of shorter duration and extent [5,6]. Emittance scans are not giving a precise enough absolute luminosity calibration, but are powerful for studying bunch effects, to monitor the stability and to measure the nonlinearity of the luminometers.

Fig. 3 on the left shows one head-on collision with crossing angle and neighbouring bunches, which experience additional deflection kicks from the bunches from the opposite beam: long-range beam-beam interactions. These kicks result in an offset of the orbit, which can be measured in the emittance scan per bunch crossing from the position of the peak of the Gaussian fit, as shown in Fig. 3 on the right. These offsets of the peak depend on the position of the bunch in the train, as the first (last) bunches of the train do not experience or experience less bunches of opposite beam on the way to (from) the interaction point than those in the middle of the bunch train.

The efficiency of the luminometers is defined to be 1 at the moment of the vdM scan. A relative change to the efficiency is monitored by evaluating the change of $\sigma_{\mathrm{vis}}$ over time as measured by 

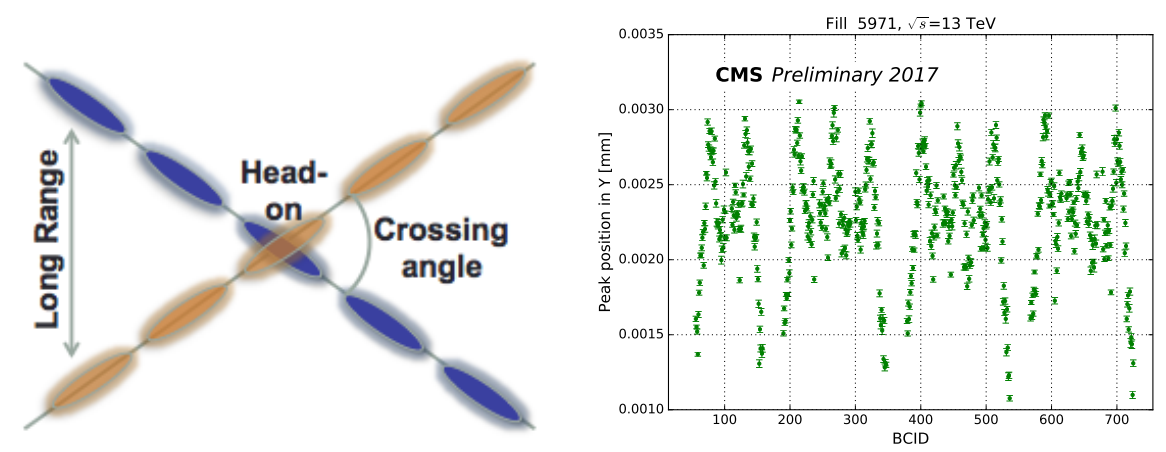

Figure 3: Sketch of the head-on collision with crossing angle (left) and the offset in the orbit due to long-range beam-beam interactions, measured as the position of the peak in the emittance scans in Y plane (right).

the emittance scans. The HF efficiency with respect to $2018 \mathrm{vdM}$ calibration fill is shown in Fig. 4 (left) for 2017 and 2018 data (based on the HFET algorithm). The observed downwards trend is due to radiation damage of the detector. The orange and red slopes correspond to the radiation damage predicted by the HCAL aging model and measured from the emittance scans, respectively.

As a wide range of a Single Bunch Instantaneous Luminosity (SBIL) is normally covered in one standard LHC fill, it is necessary to make sure that the response of the detector is linear for the whole range of luminosity. Any nonlinearity of the detector should be measured and corrected for. The nonlinearity is affecting $\sigma_{\text {vis }}$ values and can be measured from $\sigma_{\text {vis }}$ as a function of SBIL dependency. As shown in Fig. 4 (right) there is a small nonlinearity of opposite sign for leading (red), and train (blue) bunches. The nonlinearity of HFOC and HFET is of the order of $0.1-0.2 \%$ per SBIL in units of Hz/ $\mu$ b. PLT and BCM1F exhibit larger nonlinearity, of the order of $1-1.5 \%$ per unit of SBIL with same sign for leading and train bunches. The nonlinearity corrections are measured from emittance scans and applied for the whole year.
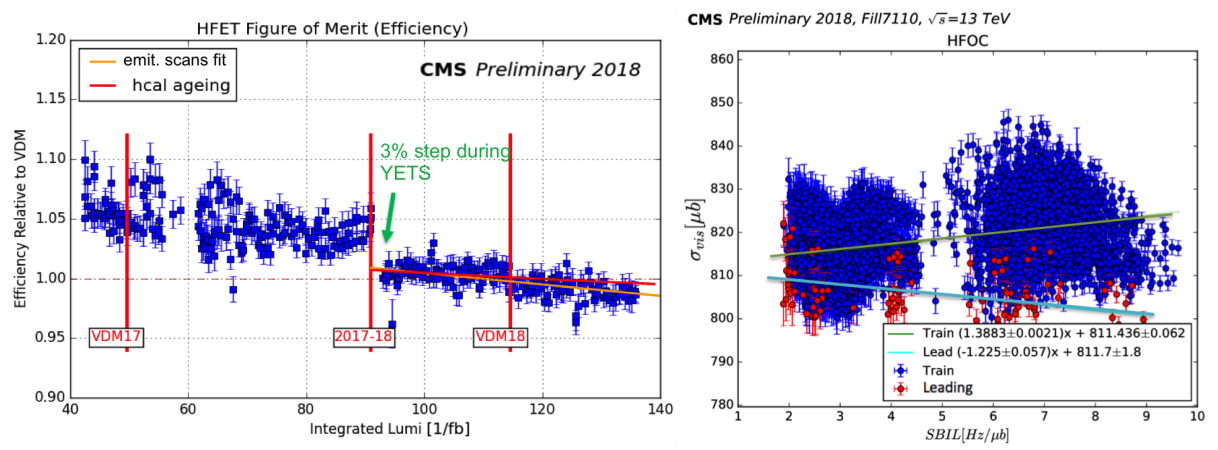

Figure 4: The HFET efficiency during 2017-2018 with respect to $\sigma_{\text {vis,vdM }}$ from the vdM scan in 2018 (left). HFOC $\sigma_{\text {vis }}$ as a function of SBIL used for the nonlinearity measurement (right).

The final luminosity uncertainty due to stability is derived from the luminosity ratios of different detectors after efficiency and nonlinearity corrections are applied. In addition to the online luminometers, PCC, RAMSES and DT data are used. RAMSES only provides orbit-integrated luminosity, but it is considered stable and linear, and is used as an additional handle for the stability measurement. As an example, measurements from three detectors are shown in Fig. 5. All detector ratios are presented and discussed in detail in Ref. [4]. The uncertainty due to stability is 
derived based primarily on the PCC/HFOC ratio and is $0.6 \%$ for 2018 . The substructure observed in the luminosity ratios is due to residual nonlinearity, originating from currently unknown sources. Based on PCC/HFOC for 2018 data residual nonlinearity of $0.2 \%$ per SBIL in units of $\mathrm{Hz} / \mu \mathrm{b}$ is measured, leading to an integration systematic uncertainty in luminosity measurement of $1.1 \%$ [4]. It is one of the largest contributions to the total luminosity uncertainty.

As an even higher instantaneous luminosity is expected in the LHC Run 3 and HL-LHC, it is of critical importance to increase the precision of the nonlinearity measurements, to improve the quality of the emittance scan technique and to derive new methods.

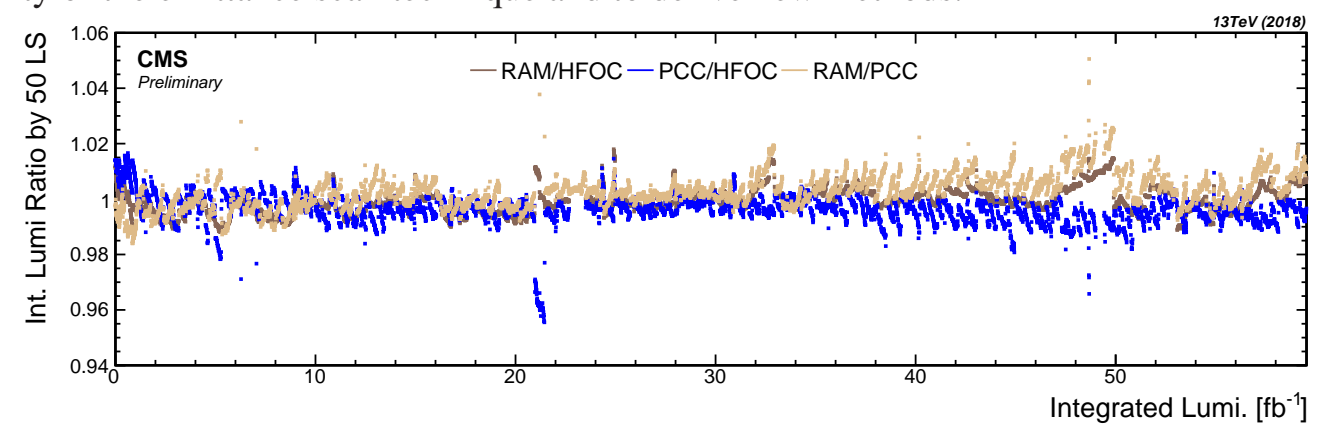

Figure 5: Luminosity ratios of the HFOC, PCC, and RAMSES subdetectors in 2018.

\section{Summary}

The readout of BRIL online luminosity detectors at $40 \mathrm{MHz}$ opened up the possibility for understanding bunch train effects, and allowed for the derivation of methods to correct for some of them. The emittance scans are powerful tools for stability and nonlinearity monitoring of the luminosity detectors. High level of confidence in the delivered luminosity is gained based on the comparison of the data from multiple CMS luminometers.

\section{References}

[1] M. Barbero et al., Design and test of the CMS pixel readout chip, Nucl. Instrum. Meth. Phys. Res., A 517 (2004) 349, DOI: 10.1016/j.nima.2003.09.043.

[2] M. Guthoff on behalf of the CMS Collaboration, Instrumentation for beam radiation and luminosity measurement in the CMS experiment using novel detector technologies, Nucl. Instrum. Meth. Phys. Res., 845 (2017) 565, DOI: 10.1016/j.nima.2016.06.028.

[3] M. Guthoff on behalf of the CMS Collaboration, The new Fast Beam Condition Monitor using polycrystalline diamond sensors for luminosity measurement at CMS, Nucl. Instrum. Methods Phys. Res., A 936 (2019) 717, DOI: 10.1016/j.nima.2018.11.071.

[4] CMS Collaboration, CMS luminosity measurement for the 2018 data-taking period at $\sqrt{s}=13 \mathrm{TeV}$, CMS-PAS-LUM-18-002, cds.cern.ch/record/2676164.

[5] M. Hostettler et. al., Luminosity scans for beam diagnostics, Phys. Rev. Accel. Beams 21, (2018) 102801, DOI: 10.1103/PhysRevAccelBeams.21.102801.

[6] O. Karacheban, P. Tsrunchev on behalf of the CMS Collaboration, Emittance scans for CMS luminosity calibration, EPJ Web of Conferences 201 (2019) 04001, DOI: $10.1051 /$ epj-conf/201920104001. 